\title{
The naturalised vascular plants of the Pilbara region, Western Australia
}

\author{
Greg Keighery \\ Department of Environment and Conservation, Locked Bag 104, Bentley Delivery Centre, Western Australia 6983, \\ Australia. Email: Greg.Keighery@dec.wa.gov.au
}

\begin{abstract}
Populations of 103 weeds are currently established in the Pilbara, comprising $6.3 \%$ of the region's flora; 19 are here newly recorded since the previous listing in 2004. Most originated in tropical Africa, America or Asia and were introduced intentionally as garden, amenity or fodder plants. Most are annual or perennial herbs with small seeds usually dispersed in soil, machinery, wind and water. Fourteen species affect the region at a landscape scale by altering fire patterns, modifying soil characteristics or competing directly with native species. Another 15 species significantly modify particular habitats such as wetlands, 6 are major threats to islands and a further 16 have potential threat to Pilbara environments.
\end{abstract}

\section{INTRODUCTION}

Since European settlement, exotic plant species (weeds) have become naturalised in Australia at the rate of about 7 to 11 species per year (Csurshes and Edwards 1998). Currently, 2739 species are naturalised in Australia (Randall 2007), which is about $19 \%$ of the flora. Even so, with a short history of European settlement, Australia is still comparatively free of weeds when compared to countries such as Britain with $32 \%$ and New Zealand with $51 \%$ of their floras naturalised exotics (Storrs and Lonsdale 1995).

Despite being recognised as a major threat to biodiversity, the naturalised vascular flora of Western Australia is still poorly documented. Approximately 1234 taxa are known, $12 \%$ of the State's total flora (Keighery and Longman 2004). The majority of these are found in the intensive land-use zone of southern Western Australia. Because of limited agricultural use, low population densities, seasonal aridity and low soil fertility, Australia's tropical regions have lower percentages of weeds. Keighery and Longman (2004) listed 86 naturalised weeds from the Pilbara bioregion (5.7\% of the total Pilbara flora), 135 (8\%) from the adjacent Carnarvon bioregion and 120 (8.6\%) from Dampierland bioregion. These figures are comparable to the Northern Territory, which has 237 species of exotics, representing $5.6 \%$ of its flora, the lowest percentage of any Australian State or Territory.

There are two key publications on Pilbara weeds-a checklist provided in Keighery and Longman (2004) and a list for the region's pastoral lands in Van Vreeswyk et al. (2004). This paper combines and revises these lists, includes the results of field survey work carried out as part of a wider biodiversity survey of the Pilbara bioregion (McKenzie et al. 2009), and provides a fuller inventory and appraisal of the conservation implications of naturalised plants in the Pilbara.

\section{METHODS}

Preparing an inventory of weeds for the Pilbara required survey methods different from those used in the elucidation of patterns in the native biota. The sample sites for the biodiversity survey were positioned in relatively undisturbed areas (McKenzie et al. 2009) and consequently sampled only weeds present at a landscape scale. The key sources for the present weed inventory were herbarium searches in combination with targeted field surveys. Records at the National Herbarium of Victoria, Melbourne, and the Western Australian Herbarium, Perth, were consulted, along with data from FloraBase (Western Australian Herbarium 1998-) and Australia's Virtual Herbarium (Australia's Virtual Herbarium 2010). Papers and reports of other recent fieldwork and taxonomic studies were also consulted. This information was supplemented by records from the Pilbara Biodiversity Survey's sample sites as well as by opportunistic records and collections made while traversing the region during this study. A specific survey was undertaken in 2006/7 to record and voucher weeds established around settlements. These are the most disturbed sites, the places where many plants first escape from cultivation. Ten new weeds for the Pilbara were located in these sites.

Accounts by explorers (Gregory and Gregory 1884), historians (Gordon 2004) and botanists (Mueller 1886) were consulted to obtain a historical 
context on the region's weeds.

Information from the above sources and other sources (see below) was used to determine, for each weed taxon, the reason the taxon was introduced and the habitats that each has affected. Further information was derived from workshops for ranking environmental weeds for the Department of Environment and Conservation (DEC) Pilbara Region, part of a Statewide program by Nature Conservation Division and DEC Science to rank weeds.

\section{RESULTS AND DISCUSSION}

The fieldwork and reviews yielded a list of 103 naturalised plants for the Pilbara, information on the reason each taxon was introduced and the main environments in which it has been found (Table 1). In comparison, 86 taxa were listed by Keighery and Longman (2004) and 44 by Van Vreeswyk (2004).

\section{Weedy status and early records}

The first unequivocal record of a weed from the region was a collection by J. Sewell from Nickol Bay in 1885. This was described as a new genus and species of lily, Elachanthera sewelliae, by Mueller (1886) but is, in fact, the weed Asparagus asparagoides (Bridal Creeper). This species was commonly cultivated in colonial homestead gardens but has not been recorded in the Pilbara since that date.

The Australian tropics are rich in pan-tropical or cosmopolitan weedy 'tramp' species. The native or naturalised status of these taxa is often queried and it is difficult to establish their status. These taxa are often treated as weeds in floras and in more general weed lists, and the exclusion of some of these from Table 1 requires justification. Eight species assigned to this category by Groves et al. (2003) are present in the Pilbara: Vachellia farnesiana, Phyla nodiflora, Helichrysum luteoalbum, Albizzia lebbek, Bidens pilosa, Echinochloa colona, Salsola kali and Persicaria lapathifolia. These taxa were most likely introduced before European settlement by Macassan fishermen from Indonesia or by birds from south-eastern Asia (Bean 2007).

The first weed records from the Pilbara were some of these cosmopolitan weedy species. Vachellia farnesiana was collected at Nickol Bay by the Gregory expedition in 1861, before the establishment of any settlements. Kodela and Tindale (2001) believe this is a pre-European introduction from Central America via the Philippines. Similarly, Phyla nodiflora was collected on the Fortescue River by J.W. Cussack in 1895 and, though the status of this species is still uncertain, it is also currently regarded as a pre-European introduction.

Helichrysum luteoalbum, collected by Alexander
Forrest on the Yule, Ashburton and Cane Rivers in 1879 (although labelled as 1870 on the collections) is currently considered either a weed or native, depending on which State or list one is following. While this species, Vachellia farnesiana and Phyla nodiflora are retained as weeds in Table 1, their status may be changed in the future.

In this paper Albizzia lebbek is considered native to the Kimberley but introduced to the Pilbara (Hussey et al. 2007). Bidens pilosa and Echinochloa colona are now considered to have been introduced to Australia (Bean 2007). In contrast, Australian plants previously called Salsola kali and considered a weed, have been shown by Borger et al. (2008) and Borger and Scott (2009) to be a native species (Salsola australis), so it is not listed in this paper. Similarly Flaveria, recorded as either F. australasica or F. trimera, is considered indigenous to Australia, having been first collected in 1802 and considered a separate taxon by Powell (1978) in his generic revision.

Persicaria lapathifolia was recorded for the Pilbara by Van Vreeswyk (2004). However, Karen Wilson (National Herbarium of New South Wales) redetermined the voucher of this record as Persicaria ?glabra (Willd.) M. Gomez, a weed that has not previously been recorded from Western Australia. I have re-examined all Persicaria collections from the Kimberley and Pilbara in the Western Australian Herbarium and re-determined these as an intermediate taxon that has been temporarily labelled as Persicaria glabra $\times$ lapathifolia. This taxon, while not referrable to a known species, is apparently a native, and will be listed as such by FloraBase. Hence, Persicaria lapathifolia is not listed in this paper.

Some plants have been shown to consist of both native and introduced populations in the Pilbara. Morrison and Scott (1996a, b) and Scott and Morrison (1996) have shown that Tribulus terrestris is a complex of native and introduced taxa. Most Pilbara records are probably the native $T$. eichlerianus, but the weedy $T$. terrestris is found around towns so this species is retained in the list. Records of Oxalis corniculata from the Hamersley Range have been re-determined as an un-named native species (Oxalis sp. Pilbara M.E. Trudgen 12275), although the weed is still present in townsites in the region (Keighery and Keighery 2008).

Similarly, Portulaca oleracea, considered by Bean (2007) to be introduced, has long been regarded as comprising native and introduced populations (Gardner 1925) in Western Australia, a view supported by a world-wide revision of the species (Danin et al. 1978) that noted 'In Australia there is a high diversity of forms which differ from those in all other parts of the world', and 'Most 
Table 1 Naturalised taxa (weeds) recorded for the Pilbara, listed against the purpose of introduction, the habitats each has affected and earliest records.

\begin{tabular}{|c|c|c|c|}
\hline FAMILY/Taxon & Purpose of introduction & Habitats affected & $\begin{array}{c}\text { Earliest record } \\
\text { (H: herbarium record; } \\
\text { L: literature record) }\end{array}$ \\
\hline $\begin{array}{l}\text { AGAVACEAE } \\
\text { Agave americana L. }\end{array}$ & ornamental & $\begin{array}{l}\text { urban (current and old } \\
\text { settlements) }\end{array}$ & $2006(\mathrm{H})$; new record \\
\hline $\begin{array}{l}\text { AIZOACEAE } \\
\text { Trianthema portulacastrum L. }\end{array}$ & unknown & $\begin{array}{l}\text { disturbed, grazed coastal, } \\
\text { alluvium, loam }\end{array}$ & $1941(\mathrm{H})$ \\
\hline $\begin{array}{l}\text { AMARANTHACEAE } \\
\text { Aerva javanica (Burm f.) } \\
\text { Juss. ex Schult. } \\
\text { Amaranthus viridis L. } \\
\text { Alternanthera pungens } \\
\text { Kunth. } \\
\text { Gomphrena celosioides Mart. } \\
\text { Pupalia lappacea (L.) Juss }\end{array}$ & $\begin{array}{l}\text { ornamental, rehabilitation, } \\
\text { fodder } \\
\text { food, fodder } \\
\text { fodder } \\
\text { unknown } \\
\text { unknown }\end{array}$ & $\begin{array}{l}\text { coastal dunes, limestone, } \\
\text { sand } \\
\text { urban } \\
\text { ruderal, highly disturbed } \\
\text { sites } \\
\text { ruderal, urban, creeklines } \\
\text { coastal }\end{array}$ & $\begin{array}{l}1920(\mathrm{~L}) \\
1947(\mathrm{H}) \\
1920(\mathrm{~L}) \\
1973(\mathrm{H}) \\
1950(\mathrm{H})\end{array}$ \\
\hline $\begin{array}{l}\text { APIACEAE } \\
\text { Cyclospermum leptophyllum } \\
\text { (Pers.) Brittan \& P.Wilson }\end{array}$ & fodder, ?unknown & urban (lawns) & $2004(\mathrm{H}) ;$ new record \\
\hline $\begin{array}{l}\text { APOCYNACEAE } \\
\text { Asclepias curassavica L. } \\
\text { Calotropis procera (Aiton) W. } \\
\text { Aiton } \\
\text { Catharanthus roseus (L.) } \\
\text { G.Don }\end{array}$ & $\begin{array}{l}\text { ornamental } \\
\text { ornamental } \\
\text { ornamental }\end{array}$ & $\begin{array}{l}\text { urban } \\
\text { alluvium } \\
\text { ruderal, urban, creeklines }\end{array}$ & $\begin{array}{l}1986(\mathrm{H}) \\
2004(\mathrm{~L}) \\
1962(\mathrm{H})\end{array}$ \\
\hline $\begin{array}{l}\text { ALOEACEAE } \\
\text { Aloe vera (L.) Burm.f. var. } \\
\text { officinalis (Forrsk.) Baker }\end{array}$ & herbal, ornamental & urban (old settlements) & $2004(\mathrm{H}) ;$ new record \\
\hline $\begin{array}{l}\text { ARECACEAE } \\
\text { Phoenix dactylifera } \mathrm{L} \\
\text { Washingtonia filifera } \\
\text { H.Wendl } \\
\text { Washingtonia robusta } \\
\text { H.Wendl. }\end{array}$ & $\begin{array}{l}\text { shade, ornamental } \\
\text { shade, ornamental } \\
\text { shade, ornamental }\end{array}$ & $\begin{array}{l}\text { riverine } \\
\text { riverine } \\
\text { riverine }\end{array}$ & $\begin{array}{l}1890(\mathrm{~L}), 1984(\mathrm{H}) \\
1890(\mathrm{~L}), 1990(\mathrm{H}) \\
2004(\mathrm{H}) ; \text { new record }\end{array}$ \\
\hline $\begin{array}{l}\text { ASPARAGACEAE } \\
\text { Asparagus asparagoides (L.) } \\
\text { Druce }\end{array}$ & ornamental & unknown & $1895(\mathrm{H})$ \\
\hline $\begin{array}{l}\text { ASPHODELACEAE } \\
\text { Asphodelus fistulosus L. }\end{array}$ & accidental & alluvium & $1979(\mathrm{H})$ \\
\hline $\begin{array}{l}\text { ASTERACEAE } \\
\text { Bidens bipinnata L. }\end{array}$ & unknown & $\begin{array}{l}\text { grazed woodlands } \\
\text { (especially Mulga), } \\
\text { creeklines, alluvium }\end{array}$ & $1962(\mathrm{H})$ \\
\hline $\begin{array}{l}\text { Bidens pilosa L. } \\
\text { Conyza bonariensis (L.) } \\
\text { Cronquist }\end{array}$ & $\begin{array}{l}\text { unknown, ?ornamental } \\
\text { accidental }\end{array}$ & $\begin{array}{l}\text { as previous } \\
\text { urban }\end{array}$ & $\begin{array}{l}1984(\mathrm{H}) \\
2001(\mathrm{H})\end{array}$ \\
\hline $\begin{array}{l}\text { Helichrysum luteoalbum L. } \\
\text { Lactuca saligna L. }\end{array}$ & $\begin{array}{l}\text { unknown } \\
\text { fodder, ?food }\end{array}$ & $\begin{array}{l}\text { riverine, wetland margins, } \\
\text { disturbed areas } \\
\text { urban }\end{array}$ & $\begin{array}{l}1879(\mathrm{H}) \\
2004(\mathrm{H}) ; \text { new record }\end{array}$ \\
\hline
\end{tabular}




\begin{tabular}{|c|c|c|c|}
\hline FAMILY/Taxon & Purpose of introduction & Habitats affected & $\begin{array}{c}\text { Earliest record } \\
\text { (H: herbarium record; } \\
\text { L: literature record) }\end{array}$ \\
\hline \multicolumn{4}{|l|}{ ASTERACEAE (continued) } \\
\hline Lactuca serriola L. & fodder, ?food & urban & $2000(\mathrm{H})$ \\
\hline Sigesbeckia orientalis L. & unknown & wetlands & $1960(\mathrm{H})$ \\
\hline Solidago canadensis L. & ornamental & urban & $1996(\mathrm{H})$ \\
\hline Sonchus oleraceus L. & accidental & wetlands & $1971(\mathrm{H})$ \\
\hline $\begin{array}{l}\text { Taraxacum officinale } \\
\text { F.H.Wigg. }\end{array}$ & accidental & urban & $2006(\mathrm{H}) ;$ new record \\
\hline Wedelia trilobata (L.) Hitchc. & ornamental & wetlands & $2006(\mathrm{H})$; new record \\
\hline \multicolumn{4}{|l|}{ BIGNONIACEAE } \\
\hline Tecoma stans (L.) Kunth & ornamental & urban areas & $2006(\mathrm{H})$; new record \\
\hline \multicolumn{4}{|l|}{ BRASSICACEAE } \\
\hline Brassica napus L. & seed & ruderal, creeklines & $2005(\mathrm{H}) ;$ new record \\
\hline Brassica tournefortii L. & contaminant & islands, coastal dunes & $2006(\mathrm{H}) ;$ new record \\
\hline Sisymbrium orientale $\mathrm{L}$. & contaminant & ruderal, urban, islands & $1996(\mathrm{H})$ \\
\hline \multicolumn{4}{|l|}{ CACTACEAE } \\
\hline Opuntia stricta (Haw.) Haw. & ornamental, edible fruit & islands, coastal sites & $1924(\mathrm{H})$ \\
\hline \multicolumn{4}{|l|}{ CHENOPODIACEAE } \\
\hline Chenopodium murale L. & salad green & urban, wetlands & $1969(\mathrm{H})$ \\
\hline \multicolumn{4}{|l|}{ CONVOLVULACEAE } \\
\hline $\begin{array}{l}\text { Merremia dissecta (Jacq.) } \\
\text { Hallier f. }\end{array}$ & ornamental & wetlands & $1962(\mathrm{H})$ \\
\hline \multicolumn{4}{|l|}{ CUCURBITACEAE } \\
\hline $\begin{array}{l}\text { Citrullus colocynthis (L.) } \\
\text { Schrad. }\end{array}$ & fruit & $\begin{array}{l}\text { ruderal, highly disturbed } \\
\text { sites }\end{array}$ & $1978(\mathrm{H})$ \\
\hline $\begin{array}{l}\text { Citrullus lanatus (Thunb.) } \\
\text { Matsum. \& Nakai }\end{array}$ & fruit & $\begin{array}{l}\text { ruderal, highly disturbed } \\
\text { sites }\end{array}$ & $1987(\mathrm{H})$ \\
\hline $\begin{array}{l}\text { Cucumis melo subsp. agrestis } \\
\text { (Naudin) Pangalo }\end{array}$ & fruit & $\begin{array}{l}\text { ruderal, highly disturbed } \\
\text { sites, creeklines }\end{array}$ & $1937(\mathrm{H})$ \\
\hline $\begin{array}{l}\text { Cucumis myriocarpus } \\
\text { Naudin }\end{array}$ & fruit & $\begin{array}{l}\text { ruderal, highly disturbed } \\
\text { sites }\end{array}$ & $2001(\mathrm{H})$ \\
\hline \multicolumn{4}{|l|}{ CYPERACEAE } \\
\hline Cyperus involucratus Poir. & ornamental & wetlands & $2006(\mathrm{H}) ;$ new record \\
\hline Cyperus polystachyos Rottb. & unknown & wetlands & $1996(\mathrm{H})$ \\
\hline Cyperus rotundus L. & unknown, ?food & wetlands & $1976(\mathrm{H})$ \\
\hline \multicolumn{4}{|l|}{ EUPHORBIACEAE } \\
\hline Euphorbia hirta L. & contaminant & urban, ruderal & $2006(\mathrm{H}) ;$ new record \\
\hline Jatropha gossypifolia L. & ornamental & grazed rangelands & $1980(\mathrm{H})$ \\
\hline Phyllanthus tenellus Roxb. & contaminant & urban (gardens) & $2006(\mathrm{H}) ;$ new record \\
\hline Ricinus communis L. & ornamental & riverine & $1995(\mathrm{H})$ \\
\hline \multicolumn{4}{|l|}{ FABACEAE } \\
\hline Albizia lebbeck (L.) Benth. & shade tree & riverine & $1890(\mathrm{~L}), 1935(\mathrm{H})$ \\
\hline Clitoria ternatea L. & ornamental & wetlands, riverine & $1969(\mathrm{H})$ \\
\hline Crotalaria juncea L. & fodder & grazed rangelands & $1995(\mathrm{H})$ \\
\hline $\begin{array}{l}\text { Desmodium scorpiurus (Sw.) } \\
\text { Desv. }\end{array}$ & fodder & grazed rangelands & $1962(\mathrm{H})$ \\
\hline Indigofera oblongifolia Forssk. & fodder & $\begin{array}{l}\text { alluvium, riverine and } \\
\text { black soils }\end{array}$ & $1982(\mathrm{H})$ \\
\hline
\end{tabular}




\begin{tabular}{|c|c|c|c|}
\hline FAMILY/Taxon & Purpose of introduction & Habitats affected & $\begin{array}{c}\text { Earliest record } \\
\text { (H: herbarium record; } \\
\text { L: literature record) }\end{array}$ \\
\hline FABACEAE (continued) & & & \\
\hline Indigofera sessiliflora DC. & fodder contaminant & grazed rangelands & $1991(\mathrm{H})$ \\
\hline $\begin{array}{l}\text { Leucaena leucocephala (Lam.) } \\
\text { de Wit }\end{array}$ & shade tree, fodder & $\begin{array}{l}\text { riverine, wetlands, } \\
\text { alluvium }\end{array}$ & $1996(\mathrm{H})$ \\
\hline Medicago polymorpha L. & fodder & $\begin{array}{l}\text { southern coastal dunes and } \\
\text { ranges }\end{array}$ & $2006(\mathrm{H})$; new record \\
\hline Parkinsonia aculeata L. & shade & riverine, alluvium & $1951(\mathrm{H})$ \\
\hline $\begin{array}{l}\text { Prosopis glandulosa } \times \\
\text { velutina Torr. \& Wooton }\end{array}$ & ornamental & riverine, alluvium & $1930(\mathrm{~L}), 1984(\mathrm{H})$ \\
\hline $\begin{array}{l}\text { Prosopis pallida (Willd.) } \\
\text { Kunth }\end{array}$ & ornamental & riverine, alluvium & $1930(\mathrm{~L}), 1936(\mathrm{H})$ \\
\hline Senna occidentalis (L.) Link & unknown & riverine, alluvium & $1975(\mathrm{H})$ \\
\hline $\begin{array}{l}\text { Stylosanthes guianensis } \\
\text { (Aubl.) Sw. }\end{array}$ & fodder & grazed rangelands & $1953(\mathrm{H})$ \\
\hline $\begin{array}{l}\text { Stylosanthes hamata (L.) } \\
\text { Taub }\end{array}$ & fodder & grazed rangelands & $1997(\mathrm{H})$ \\
\hline Tamarindus indica $\mathrm{L}$. & shade tree, fruit & urban & $1932(\mathrm{H})$ \\
\hline $\begin{array}{l}\text { Vachellia farnesiana (L.) } \\
\text { Wight \& Arn. }\end{array}$ & unknown & $\begin{array}{l}\text { many habitats, especially } \\
\text { alluvium, grazed } \\
\text { rangelands }\end{array}$ & $1861(\mathrm{H})$ \\
\hline MALVACEAE & & & \\
\hline Gossypium hirsutum L. & seeds, ornamental & urban & $1996(\mathrm{H})$ \\
\hline $\begin{array}{l}\text { Malvastrum americanum (L.) } \\
\text { Torr. }\end{array}$ & unknown & many habitats & $1938(\mathrm{H})$ \\
\hline $\begin{array}{l}\text { Malvastrum } \\
\text { coromandelianum (L.) Garcke }\end{array}$ & unknown & many habitats & $1982(\mathrm{H})$ \\
\hline Melochia pyramidata L. & unknown & $\begin{array}{l}\text { many habitats, especially } \\
\text { alluvium }\end{array}$ & $1931(\mathrm{H})$ \\
\hline MORINGACEAE & & & \\
\hline Moringa oleifolia Lam. & ornamental & creeklines & $1973(\mathrm{H})$ \\
\hline NYMPHAEACEAE & & & \\
\hline $\begin{array}{l}\text { Nymphaea macrosperma } \\
\text { Merr. \& L.Perry }\end{array}$ & ornamental & riverine pools & 1890 (L), $1958(\mathrm{H})$ \\
\hline OXALIDACEAE & & & \\
\hline Oxalis corniculata $\mathrm{L}$. & lawn weed & urban & $2006(\mathrm{H})$; new record \\
\hline PAPAVERACEAE & & & \\
\hline $\begin{array}{l}\text { Argemone ochroleuca Sweet } \\
\text { subsp. ochroleuca }\end{array}$ & ornamental & riverine, alluvium & $1916(\mathrm{H})$ \\
\hline PASSIFLORACEAE & & & \\
\hline $\begin{array}{l}\text { Passiflora foetida var. hispida } \\
\text { (Triana et Planch.) Killip }\end{array}$ & unknown & $\begin{array}{l}\text { coastal dunes, islands, } \\
\text { rocky sites, riverine }\end{array}$ & $1946(\mathrm{H})$ \\
\hline POACEAE & & & \\
\hline Andropogon gayanus Kunth & fodder & riverine, alluvium & $1953(\mathrm{H})$ \\
\hline Arundo donax L. & ornamental & riverine & $1890(\mathrm{~L}), 1996(\mathrm{H})$ \\
\hline Avena barbata $\mathrm{L}$. & fodder & $\begin{array}{l}\text { southern coastal dunes, } \\
\text { islands }\end{array}$ & $2006(\mathrm{H}) ;$ new record \\
\hline Cenchrus ciliaris L. & fodder & $\begin{array}{l}\text { many habitats, especially } \\
\text { coastal dunes }\end{array}$ & $1900(\mathrm{~L}), 1922(\mathrm{H})$ \\
\hline Cenchrus echinatus L. & fodder & riverine, alluvium & $1900(\mathrm{~L}), 1983(\mathrm{H})$ \\
\hline
\end{tabular}




\begin{tabular}{|c|c|c|c|}
\hline FAMILY/Taxon & Purpose of introduction & Habitats affected & $\begin{array}{c}\text { Earliest record } \\
\text { (H: herbarium record; } \\
\text { L: literature record) }\end{array}$ \\
\hline POACEAE (continued) & & & \\
\hline Cenchrus setiger Vahl. & fodder & riverine, alluvium, ruderal & 1900 (L), $1929(\mathrm{H})$ \\
\hline Chloris barbata Sw. & unknown & riverine, alluvium, ruderal & $1979(\mathrm{H})$ \\
\hline Chloris virgata Sw. & fodder & riverine, alluvium, ruderal & $1953(\mathrm{H})$ \\
\hline Cynodon dactylon (L.) Pers. & ornamental & wetlands, urban & $1941(\mathrm{H})$ \\
\hline $\begin{array}{l}\text { Dichanthium annulatum } \\
\text { (Forrsk.) Stapf }\end{array}$ & fodder & wetlands & $2001(\mathrm{H})$ \\
\hline $\begin{array}{l}\text { Digitaria ciliaris (Retz.) } \\
\text { Koeler }\end{array}$ & ?fodder, contaminant & riverine, alluvium, ruderal & $1992(\mathrm{H})$ \\
\hline Echinochloa colona (L.) Link & fodder, ?birdseed & wetlands & $1941(\mathrm{H})$ \\
\hline Eragrostis minor Host. & ?fodder, contaminant & ruderal & $1988(\mathrm{H})$ \\
\hline Eragrostis pilosa (L.) Beauv. & ?fodder, contaminant & ruderal & $2002(\mathrm{H})$ \\
\hline Lamarckia aurea (L.) Moench & ornamental, fodder & alluvium & $1953(\mathrm{H})$ \\
\hline $\begin{array}{l}\text { Leptochloa fusca subsp. } \\
\text { uninervia (J.Presl) N.Snow }\end{array}$ & fodder, rehabilitation & wetlands, alluvium & $1958(\mathrm{H})$ \\
\hline Melinis repens (Willd.) Zizka & ornamental, fodder & ruderal, rangelands & $2006(\mathrm{H})$; new record \\
\hline $\begin{array}{l}\text { Paspalum fasciculatum } \\
\text { Fluegge }\end{array}$ & fodder & wetlands & $1953(\mathrm{H})$ \\
\hline $\begin{array}{l}\text { Pennisetum setaceum } \\
\text { (Forrsk.) Chiov }\end{array}$ & ornamental & $\begin{array}{l}\text { coastal dunes, southern } \\
\text { rangelands }\end{array}$ & $1953(\mathrm{H})$ \\
\hline $\begin{array}{l}\text { Polypogon monspeliensis (L.) } \\
\text { Desv. }\end{array}$ & fodder & $\begin{array}{l}\text { fresh and brackish } \\
\text { wetlands }\end{array}$ & $1995(\mathrm{H})$ \\
\hline Setaria italica (L.) P.Beauv. & bird seed, fodder & ?localised, urban & $1898(\mathrm{H})$ \\
\hline $\begin{array}{l}\text { Setaria sphacelata } \\
\text { (Schumach.) Stapf. \& } \\
\text { C.E.Hubb. }\end{array}$ & fodder & ?localised, urban & $1953(\mathrm{H})$ \\
\hline Setaria verticillata (L.) P.Beauv. & fodder & ?localised, urban & $1937(\mathrm{H})$ \\
\hline $\begin{array}{l}\text { POLYGONACEAE } \\
\text { Acetosa vesicaria (L.) A.Love }\end{array}$ & ornamental, rehabilitation & $\begin{array}{l}\text { potentially widespread on } \\
\text { alluvium, clay and loam soils }\end{array}$ & $1971(\mathrm{H})$ \\
\hline $\begin{array}{l}\text { PORTULACACEAE } \\
\text { Portulaca oleracea L. }\end{array}$ & unknown (native and weed) & urban, ruderal & $2006(\mathrm{H}) ;$ new record \\
\hline $\begin{array}{l}\text { PTERIDIACEAE } \\
\text { Ceratopteris thalictroides (L.) } \\
\text { Brongn. }\end{array}$ & ornamental & riverine, river pools & $1890(\mathrm{~L}), 1932(\mathrm{H})$ \\
\hline $\begin{array}{l}\text { SALVINIACEAE } \\
\text { Salvinia molesta D.S.Mitch. }\end{array}$ & ornamental & riverine, river pools & $1986(\mathrm{H})$ \\
\hline $\begin{array}{l}\text { SOLANACEAE } \\
\text { Datura leichhardtii Benth. } \\
\text { Datura metel L. } \\
\text { Physalis angulata L. } \\
\text { Solanum nigrum L. }\end{array}$ & $\begin{array}{l}\text { unknown } \\
\text { ornamental } \\
\text { fruit, unknown } \\
\text { fruit }\end{array}$ & $\begin{array}{l}\text { creeklines } \\
\text { urban } \\
\text { creeklines } \\
\text { urban, wetlands, riverine }\end{array}$ & $\begin{array}{l}1941(\mathrm{H}) \\
1988(\mathrm{H}) \\
1995(\mathrm{H}) \\
1997(\mathrm{H})\end{array}$ \\
\hline $\begin{array}{l}\text { TAMARICACEAE } \\
\text { Tamarix aphylla L. }\end{array}$ & shade & riverine & $2006(\mathrm{H}) ;$ new record \\
\hline $\begin{array}{l}\text { VERBENACEAE } \\
\text { Phyla nodiflora (L.) Greene } \\
\text { var. nodiflora }\end{array}$ & ornamental, lawns & alluvium, riverine & $1895(\mathrm{H})$ \\
\hline $\begin{array}{l}\text { ZYGOPHYLLACEAE } \\
\text { Tribulus terrestris L. }\end{array}$ & unknown/contaminant & ruderal, grazed rangelands & $1991(\mathrm{H})$ \\
\hline
\end{tabular}


specimens that we have seen from different parts of Australia and New Zealand do not agree with our description of the subspecies from other parts of the world'. Populations collected in 2006 in towns and along roadsides in the Pilbara correspond to the inbreeding, small-flowered weedy bio-type of southern Western Australia.

Taxonomic re-arrangements have changed or removed other records. Sonchus asper was recorded for the Pilbara by Van Vreeswyk (2004) but, at that time, the specimens identified as this species included the native Sonchus hydrophilus. All Pilbara records are the native species. The record of Digitaria sanguinalis in Van Vreeswyk (2004) is now considered to be $D$. ciliaris. The record of Zizyphus mauritiana in Van Vreeswyk (2004) is from Onslow, which is outside the Pilbara. Conversely, Calotropis procera, recorded by Longbottom (2004) as established along the De Grey River system, is not recorded elsewhere in the Pilbara. This species is highly invasive of black soil plains in Western Australia and the Northern Territory and is being actively controlled in the Pilbara.

A number of introduced species are not considered to have become naturalised in the Pilbara. These include non-persistent species of refuse sites. Just after the wet season, we recorded vigorous stands of many cucurbit vines along rivers adjacent to the North West Coastal Highway. The stands had sprouted from material buried by visitors during the previous dry season and included Cucumis sativus L. (Cucumber) (G. Keighery 17365) from India; Cucurbita pepo (L.) Dumort. (Squash) (G. Keighery 17362) from Africa; Cucumis melo L. subsp. melo (Melon) (G. Keighery 17364) from America; Curcubita maxima Duschene ex Lam. (Pumpkin) (G. Keighery 17363) from South America; and Citrillus lanatus var. vulgaris (Schrad.) Fursa (Watermelon) (G. Keighery 17366) from Africa. It is unlikely that these annuals would re-seed and they should be regarded as having established temporary populations in the region.

Suckering stands of Bougainvillea (Bougainvillea glabra Choisy) were also recorded as long-persistent plants on several abandoned stations, but these are not naturalised. A stand of the cactus Hylocereus undulatus (Haw.) Brittan \& Rose (Night-blooming Cereus) was recorded on the rubbish tip at Cane River Station but has been eradicated.

At workshops held in 2009 to prioritise environmental weeds in the Pilbara, ten weeds in mainly urban situations were noted that require collections for determination before being added to the Pilbara weed list:

- Cardiospermum halicacabum L. var. halicacabum (Sapindaceae), Small Balloon Creeper Vine, Karratha gardens;
- Euphorbia tirucalli L. (Euphorbiacaeae), Finger Tree or Naked Lady, eradicated from Pippingarra Station;

- Nerium oleander L. (Apocynaceae), Oleander, Dampier gardens;

- Celosia argentea L. (Amaranthaceae), Cockscomb, Karratha gardens;

- Ipomoea quamoclit L. (Convolvulaceae), Cupid's Flower, Port Hedland;

- Crotalaria juncea L. (Fabaceae), Sun Hemp, Port Hedland;

- Gnaphalium polycaulon Pers. (Asteraceae), Western Cudweed, Karratha;

- Cenchrus incertus M.A. Curtis (Poaceae), Spiny Burrgrass, Port Hedland.

Participants reported that either Paspalum distichum L. (Poaceae) or P. vaginatum Sw., both known as Saltwater Couch, is present in wetlands in the Pilbara.

There are also weeds noted in consultancy reports that are not supported by voucher collections in the Western Australian Herbarium, or that are listed rarely or listed as eradicated:

- Bassia scoparia L. (Chenopodiaceae), Kochia, has been recorded from Marandoo (although it was in the nursery) in Mattiske (1992). Biota (2009) again listed this species, but its present status is unknown.

- Cenchrus biflorus Roxb. (Poaceae), Gallon's Curse, was recorded at Millstream, subsequently eradicated. However, sight records by S. van Leeuwen indicate that this species is still being introduced into the Pilbara on vehicles.

- Chloris gayana Kunth (Poaceae), Rhodes Grass, was growing in rehabilitation areas and along creek lines in the Shay Gap/Yarrie area (Strategen 2006, BHP 2009). However, sight records by S. van Leeuwen around Karratha and Tom Price, indicate that this species is also still being introduced into the Pilbara on vehicles.

- Emex australis Steinh. (Polygonaceae), Doublegee, was recorded at Yandicoogina by Mattiske (1995).

- Euphorbia peplus L. (Euphorbiaceae), Petty Spurge, has been recorded from Marandoo (Biota 2009).

- Lawsonia inermis L. (Lythraceae), Henna, was recorded around Dampier by V. Long (pers. comm.).

- Ocimum basilicum L. (Lamiaceae), Sweet Basil, was recorded from a creekline east of the Bungaroo Road (Biota 2006).

- Sisymbrium irio L. (Brassicaceae), London Rocket, 
was recorded in Marillana Creek (Biota 2004).

- Vitex trifolia var. subtrisecta (Kuntze) Mold. (Lamiaceae), Beach Vitex, was recorded growing in disturbed coastal dune habitat at Cape Lambert (Biota 2008).

- Vinca major L. (Apocynaceae), Blue Periwinkle, recorded from a creekline east of the Mesa A transport corridor (Biota 2006).

Finally, neither Borassus sp. (Borassus Palm), which has been eradicated, nor Helianthus annuus L. (Sunflower) appears in Table 1. Sporadic sightings of individual plants of $H$. annuus have been made near Pannawonica and Dampier, but this species may eventually become established in the southern coastal areas of the Pilbara. Of those taxa not yet definitely recorded as weeds in the region, it would appear that three-Chloris gayana, Cenchrus biflorus and Vitex trifolia var. subtrisecta-have the potential become naturalised members of the flora of the Pilbara.

\section{History of introductions}

European settlement started in the Pilbara in 1863 with the establishment of the first pastoral lease, De Grey Station, followed by Cossack port and Roebourne townsite in 1864 (Withnell 1936). No doubt plants were introduced from this time, since many early publications and photographs show established kitchen and ornamental gardens at pastoral homesteads.

A good example of this process is from Millstream Station $\left(21^{\circ} 35^{\prime} 25^{\prime \prime} \mathrm{S}, 117^{\circ} 04^{\prime} 05^{\prime \prime} \mathrm{E}\right)$, first selected in 1864 by Alex McRae and Trevor McKenzie. It was operated by them until 1879 when the lease was purchased by Walter Padbury and William Loton (Withnell Taylor 2002) who ran it until 1914. Numerous exotic species were introduced around the homestead and along the river to add shade and beautification. From photographs illustrated in Gordon (2004, p. 73), these included Tamarisk (Tamarix aphylla) and Albizia (Albizia lebbek). Cotton Palm (Washingtonia filifera), Date Palm (Phoenix dactylifera), Bamboo (Arundo donax), Borassus Palm (Borassus aethiopica?), Yellow Oleander (Thevetia peruviana) and Oleander (Nerium oleander) were planted along the Fortescue River. Several ornamental aquatics, including Waterlily (Nymphaea macrosperma) and the Indian Water Fern (Ceratopteris thalictroides), were planted in Chinderwarriner Pool adjacent to the homestead.

By 2007, many of these had disappeared, and only a single Borassus Palm remained (Anon. 2007), which has since been removed. However, the Date Palm, Cotton Palm, Waterlily and Indian Water Fern have become major local weeds. The first herbarium record of the Water Fern dates from a collection made at Millstream (21 $35^{\prime} 25^{\prime \prime} S$, $\left.117^{\circ} 04^{\prime} 05^{\prime \prime} \mathrm{E}\right)$ by Charles Gardner in August 1932, and the first Waterlily specimen was collected by K. Vollprecht from Crystal Pool (21 $\left.35^{\prime} 23^{\prime \prime} \mathrm{S}, 117^{\circ} 03^{\prime} 59^{\prime \prime} \mathrm{E}\right)$ between 1958 and 1960. The Palms, being rather difficult to process as herbarium material, were not collected until much later: Date Palm by Alex George in March 1962, and Cotton Palm by Estelle Leyland in July 1990. This illustrates a gap of up to 100 years between introduction or establishment and herbarium documentation.

Table 1 lists the mode of entry into Australia of the weeds recorded for the Pilbara. A series of introduction categories (Lazarides et al. 1997) predominate:

- 39 taxa for fodder or food;

- 36 taxa as ornamental garden plants; and

- 7 accidental.

The origins of introduction of 22 taxa are unknown.

The figures show that most taxa (over 70\%) were deliberately introduced for horticulture or agriculture, a proportion that is typical for weeds in general. However, the timing, causes and methods of introduction to the Pilbara were often different from the original reasons for introduction and are difficult to establish. For example, Buffel Grass (Cenchrus ciliaris) was noted by Hennig (2004) to have been introduced to the Port Hedland area in the saddle padding for Afghan camels in the 1920s. It then became established on the town common and was subsequently spread as a pasture species. However, it is understood that this species was introduced into Australia elsewhere as a pasture species. Other authors also refer to different reasons for its introduction to the Pilbara. For example Van Vreeswyk (2004) noted that Buffel Grass was introduced by pastoralists to the delta of the Yule River in the early 1900s. Petheram and Kok (1983) noted that Aerva javanica and Calotropis procera were also used as padding in camel saddles and were introduced to the Pilbara in a similar manner, a statement supported by the earliest collection of Aerva from the Pilbara in 1920. Aerva javanica was subsequently used widely for revegetation in the Ord and Fitzroy basins. At the same time, Birdwood Grass (Cenchrus setiger) was introduced for fodder to Mundabullangana Station from a sample of seed sent from India (Van Vreeswyk 2004).

Table 1 gives the earliest known dates of recording of the presence of weeds in the Pilbara. Introductions have been documented at a steady rate since settlement began in 1863. As noted above, in the past there has been a significant lag phase between introduction and the collection of a herbarium specimen to indicate that a species has 
naturalised. Most records before 1960 were made at major ports (most collectors also visited these) and pastoral station homesteads. Currently, most new records are found around towns and roadsides. The number of introductions of new weeds has not slowed over the reporting period (1861-2006), but the rate of detection has probably increased in the past decade. Herbarium collections are becoming a more accurate measure of the date of naturalisation.

Thus, some species have been introduced to the Pilbara several times, and for various reasons, since the original introductions to Australia. The main reasons in the past and present, however, remain for gardens, in contaminated soil (machinery, roads) and as pastoral plants.

\section{Richness and biogeographical origins}

After considering the revisions and changes noted above, a total of 103 weed taxa are currently established in the Pilbara (Table 1). The native flora of the Pilbara comprises 1521 taxa (Western Australian Herbarium 1998-, accessed 22 April 2010), hence of a total flora of 1624 taxa, $6.34 \%$ are naturalised, which indicates that the region is comparatively weed-free in terms of species of naturalised flora, although perhaps not in number of plants or extent of some species, such as Buffel Grass. Growth forms of naturalised species are mainly annual herbs (56) or perennial herbs (23), with a few trees (11), shrubs (12) and vines (2). There are 31 families represented in the weed flora, Poaceae (23), Fabaceae (16) and Asteraceae (11) having the highest representation.

Pilbara weeds originate mainly from areas with similar climates (Table 2), especially the Americas (34 species), Asia (27 species) and Africa (11 species). There is a smaller element of temperate weeds originating from Europe (10) and the Mediterranean to Western Asia (6), found mainly around towns of the southern and coastal Pilbara. Finally, there is a cosmopolitan element of nine species.

\section{Taxa that are current or potential threats to the Pilbara}

The Pilbara is a semi-arid region characterised by rugged terrain, external drainage by fresh permanent and seasonal rivers, coastal plains and complex patterns of alluvial plains created by the rivers. The large, fresh rivers and wetlands are a unique feature of the Pilbara compared with the desert regions of Western Australia, and any weed that affects these values adversely is significant. Examples of some of these are highlighted below.

The Pilbara remains relatively weed-free and quarantine measures at the national borders contribute to keeping out potential weeds from
Table 2 Geographic origin of Pilbara weeds.

\begin{tabular}{l|c}
\hline Geographic area of origin & Number of taxa \\
\hline AMERICA & 8 \\
North America & 9 \\
South America & 17 \\
Tropical America & \\
ASIA & 6 \\
South West Asia & 5 \\
Tropical Asia to Australia & 17 \\
Africa to Asia & \\
AFRICA & 8 \\
Tropical Africa & 3 \\
Southern Africa & \\
EUROPE & 6 \\
Mediterranean & 10 \\
Europe & \\
COSMOPOLITAN & \\
Tropical & \\
Cosmopolitan & \\
\hline
\end{tabular}

overseas. However, many weedy species that could pose a significant threat to biodiversity in the region are already present elsewhere in Australia and so cannot be addressed by national quarantine. Some of these occur in low numbers in the Pilbara. Martin et al. (2006) listed 622 non-native naturalised species from Australian rangelands, of which 160 species $(26 \%)$ were considered to pose a threat to rangeland biodiversity. Subsequently, Grice and Martin (2006) listed 92 weeds as posing the greatest actual or potential threat to biodiversity in the Australian rangelands. They cited 20 as major threats to the Pilbara, of which 9 are here recorded as already present (Table 1) : Calotropis procera, Cenchrus ciliaris, Jatropha gossypifolia, Leucaena leucocephala, Opuntia spp., Phyla spp., Prosopis spp., Salvinia molesta and Senna occidentalis. Two others listed listed by Grice and Martin (2006), but not specifically identified as a threat to the Pilbara, are now known to be present: Agave americana and Asphodelus fistulosus.

A further eleven were listed as having the potential to invade the Pilbara Bioregion but have not yet been recorded, these being: Acacia nilotica, Bryophyllum daigremontianum $\times$ delagoense, Carrichtera annua, Lepidium didymum, Cryptostegia grandiflora, Eichhornia crassipes, Lantana camara, Pennisetum polystachion, Sida acuta, Sida rhombifolia 
and Zizyphus mauritiana. In addition to those listed by Grice and Martin (2006), the following should be considered potential threats: Brassica tournefortii, Cylindropuntia spp., Melinis repens, Zizyphus mauritiana and Xanthium strumarium. These can be addressed through a combination of listing under the State Biosecurity Legislation, effective local quarantine to prevent spread to the region, and eradication of new populations before widespread establishment. Several are already Declared Plants under the Agriculture and Related Resources Protection Act 1976.

While it is not possible to predict accurately all Pilbara habitats that may be affected by many of these species, for some it is clear. For example, Xanthium strumarium (Noogoora Burr) is highly likely to invade watercourses and alluvial flood plains. Prevention of invasion or eradication of small populations of these weeds is a highly costbeneficial option and should remain a focus of control efforts.

Grice and Martin (2006) noted that, in general, invasive plants reduce the diversity and abundance of native plants in invaded habitats. In this context, eleven species have been briefly discussed by McKenzie et al. (2009): Acetosa vesicaria, Aerva javanica, Cenchrus ciliaris, C. setiger, Calotropis procera, Leucaena leucocephala, Phoenix dactylifera, Prosopis spp., Tamarix aphylla and Zizyphus mauritiana. Table 1 lists weeds of the Pilbara, the purpose of introduction, the habitats they currently occupy and the earliest known record. Three groups warrant special mention-1, those that affect many habitats across the landscape, 2, those that affect specific habitats and 3 , those on the islands.

\section{Landscape-scale weeds (14 taxa)}

These are weeds able to grow in many habitats, often becoming dominant. Generally they alter the habitats they invade by changing soil chemistry, fire regimes, competition or a combination of these. The following 10 taxa from this category are the major weeds of the Pilbara: Acetosa vesicaria, Aerva javanica, Argemone ochroleuca, Cenchrus ciliaris, C. setiger, Chloris virgata, Indigofera oblongifolia, Malvastrum americanum, Prosopis pallida and Prosopsis glandulosa $\times$ velutina. The key source for this determination is Van Vreeswyk (2004). Of the 12,445 traverses that Van Vreeswyk et al. (2004) scored, $14 \%$ contained weeds. The most common species was Cenchrus ciliaris, recorded on $12 \%$ of transects and estimated to dominate the coastal plains and alluvial floodplains of the area, or some $3,434 \mathrm{~km}^{2}$ of the Pilbara. Aerva javanica, Malvastrum americanum, Acetosa vesicaria, Chloris virgata, Indigofera oblongifolia, Prosopis spp. and Argemone ochroleuca were also recorded at several transects in a range of habitats.
From surveys for this paper, Cenchrus setiger, the two Bidens spp. and two Stylosanthes spp. were found to be increasingly widespread weeds of many habitats and are added to the list of landscape-scale weeds.

\section{Weeds of specific habitats (15 taxa)}

Fifteen of these species invade and severely affect localised habitats, especially the unique rivers and wetlands of the Pilbara which are invaded by the following taxa:

- Phoenix dactylifera, Washingtonia filifera, Washingtonia robusta, Tamarix aphylla, Ricinus communis, Clitoria ternatea, Parkinsonia aculeata, Leucaena leucocephala and Leptochloa fusca subsp. uninervia tolerate waterlogging/flooding and are major weeds of the river banks and courses;

- Ceratopteris thalictroides, Salvinia molesta and Nymphaea macrosperma are major weeds of water bodies;

- Polypogon monspeliensis is a salt-tolerant species that could be a major threat to the Fortescue Marsh;

- Paspalum fasciculatum is a potentially serious threat to the diverse freshwater claypans; and

- Cynodon dactylon, which is already regarded as the most serious weed of arid wetlands in central Australia (Duguid et al. 2002).

\section{Weeds of the islands (6 taxa)}

Although not included in the Pilbara Biodiversity Survey, the islands off the Pilbara coast contain a set of weeds that should be mentioned. These islands have unique biodiversity values, especially for their fauna, and are potentially at risk from the following weeds: Opuntia stricta, Eragrostis curvula, Hyparrhenia hirta and Melinis repens. Based on experience elsewhere, two others could become significant on the islands-Jatropha gossypifolia and Asphodelus fistulosus - which may invade alluvial, species-rich herblands.

\section{Current control measures}

Weed management in the Pilbara has progressed from documentation to active management aimed at reducing the establishment of new weeds, eliminating potential weeds, removing the threat to biodiversity of certain weeds, and planning to reduce the threat of landscape-scale weeds.

An active program is underway to reduce the establishment of new weeds, by replacing invasive exotics in gardens (Department of Environment and Conservation 2009). Most major mining developments now include weed management plans (e.g. Strategen 2006; Fortescue Metals Group 2009). These plans aim to prevent the introduction 
of new weeds through mining operations, control existing weeds on mining leases, and reduce their impact and presence on rehabilitation areas. Both Cotton and Date Palms are under active control at Millstream (Kendrick 2005), as are the two major invasive aquatics, Ceratopteris thalictroides and Nymphaea macrosperma. Prickly Pear has been eradicated from the Dampier Archipelago. Attempts are under way to control the infestation of Calotropis on the De Grey River, with cost-effective methods devised by the Western Australian Department of Agriculture and Food (A. Reeves, pers. comm.).

Management plans are being prepared for several major weeds, including Acetosa (Anthony and Dixon 2006), Parkinsonia (Department of Water 2008) and Prosopis (Anderson et al. 2006; van Klinken et al. 2007; Land and Water Australia 2008b). Attempts are being made to limit the spread of Buffel Grass onto offshore islands (Dixon et al. 2002) and to prepare research and management programs on this weed (Friedel et al. 2007; Land and Water Australia 2008a).

A potential future risk is posed by pastoral diversification initiatives, especially when this involves growing crops for hay, pasture or biofuel. Many species grown for bio-fuel are known environmental weeds, some very serious and aggressive. Many proposed pasture species are weedy grasses that have the potential to become environmental weeds if not managed carefully, especially in riparian areas.

\section{CONCLUSIONS}

The weed flora of the Pilbara (103 taxa) is relatively small, being just over $6 \%$ of Pilbara's total flora. Compared with the 2004 listing of 84 weeds (Keighery and Longman 2004), this is an increase of 19 taxa $(20 \%)$ in six years. While most likely an overestimate due to changes in designation of taxa (weed status and taxonomy), this increase is of concern. As most species were introduced intentionally as garden, amenity or fodder plants, there is a need for continuing quarantine to limit further introductions. There is also a need to keep track of the taxa that are currently present in small numbers but are considered to have the potential to expand.

Of the 103 weeds, 14 species affect the region at a landscape scale, altering fire patterns, modifying soil characteristics, or competing directly with native species. Another 21 significantly affect particular habitats, especially wetlands and islands. It is essential that management actions/plans be developed to focus on these weeds. Taxa that are currently present in small numbers but have the known or predicted potential to expand are another priority for control measures. The actions by DEC to rank weeds for management actions are endorsed and should continue, as are current control actions.

\section{ACKNOWLEDGEMENTS}

The Curators and staff of the National Herbarium of Victoria and the Western Australian Herbarium allowed access to collections under their care. Pilbara weed prioritisation workshops were funded by Nature Conservation Division and the weed survey by the Department of Environment and Conservation. Vicki Long, Steve van Leeuwen and Michael Lyons made useful comments and valuable additions to drafts of the paper.

\section{REFERENCES}

Anderson, L.J., Van Klinken, R.D., Parr, R.J., Climas, R. and Barton, D. (2006). Integrated management of hybrid mesquite: a collaborative fight against one of Australia's worst woody weeds (pp. 239-242). In: Preston, C., Watts, J.H. and Crossman, N.D. (eds), Managing weeds in a changing climate: 15th Australian Weeds Conference Papers \& Proceedings, Adelaide, 24-28 September 2006, Weed Management Society of South Australia: Adelaide, Australia.

Anonymous. (2007). Millstream-Chichester National Park and Mungaroona Range Nature Reserve, draft management plan. Millstream Park Council, Department of Environment and Conservation, Conservation Commission of Western Australia: Kensington, Australia.

Anthony, J.M. and Dixon, I.R. (2006). Research and development for integrated control of Acetosa vesicaria (Ruby Dock) in the Pilbara Region of Western Australia. Botanic Gardens and Parks Authority: Perth, Australia.

Australia's Virtual Herbarium (2010). http:/ /www.ersa. deu.au/avh (accessed 9 April 2010).

Bean, A.R. (2007). A new system for determining which plant species are indigenous in Australia. Australian Systematic Botany 20: 1-43.

BHP Billiton (2009). Goldsworthy iron ore mining operations. weed management plan, revision 2. BHP Limited: Melbourne, Australia.

Biota (2004). Yandicoogina expansion vegetation and flora survey. Report prepared for Hamersley Iron, Australia.

Biota (2006). A vegetation and flora survey of the proposed Mesa A transport corridor, Warramboo Deposit and Yarraloola Borefield. Report prepared for Robe River Associates.

Biota (2008). Cape Lambert Port B development: flora and vegetation survey. Report prepared for Rio Tinto Australia.

Biota (2009). Marandoo Phase 2: flora and vegetation survey. Report prepared for Rio Tinto Australia.

Borger, C.P.D., Yan, G., Scott, J.K., Walsh, M. and Powles, S. (2008). Salsola tragus or S. australis (Chenopodiaceae) in Australia - untangling the taxonomic confusion through random amplified polymorphism (RAMP) and cytological analysis. 
Australian Journal of Botany 56: 600-608.

Borger, C.P.D. and Scott, J.K. (2009). The biology of Australian weeds, 55, Salsola australis R.Br. Plant Protection Quarterly 24: 126-137.

Csurshes, S. and Edwards, R. (1998). Potential environmental weeds in Australia. Environment Australia: Canberra, Australia.

Danin, A., Barker, I. and Barker, H. (1978) Cytogeography and taxonomy of the Portulaca oleracea L. polyploid complex. Israel Journal of Botany 27: 177-211.

Department of Environment and Conservation (2009). What to grow instead of: (Cotton Palms, Date Palms, Leucaena, Lippia and Oleander). Department of Environment and Conservation: Karratha, Australia.

Department of Water (2008). A field guide for eradicating Parkinsonia in the Fortescue River Area. Western Australian Department of Water: Perth, Australia.

Dixon, I.R., Dixon, K.W. and Barrett, M. (2002). Eradication of Buffel Grass (Cenchrus ciliaris) on Airlie Island, Pilbara coast, Western Australia (pp 92-101). In: Veitch, C.R. and Clout, M.N. (eds), Turning the tide: the eradication of invasive species. IUCN ISSG: Gland, Switzerland.

Duguid, A., Barnetson, J., Clifford, B., Pavey, C., Albrecht, D., Risler, J. and McNellie, M. (2002). Wetlands in the arid Northern Territory. A report to Environment Australia on the inventory and significance of wetlands in the arid NT. Parks and Wildlife Commission of the Northern Territory: Alice Springs, Australia.

Fortescue Metals Group (2009). Pilbara iron ore and infrastructure project: weed hygiene and management plan. Fortescue Metals Group: East Perth, Australia.

Friedel, M., Bastin, G., Brock, C., Butler, D., Clarke, A., Eyre, T., Fox, J., Grice, A., van Leeuwen, S., Pitt, J., Puckey, H. and Smith, A. (2007). Developing a research agenda for the distribution and rate of spread of buffel grass (Cenchrus ciliaris) and identification of landscapes and biodiversity assets at most risk from invasion. Report to the Department of Environment and Water: Canberra, Australia.

Gardner, C.A. (1925). List of the naturalised plants of extra-tropical Western Australia. Journal of the Royal Society of Western Australia 11: 69-80.

Gordon, D.L. (2004). The West Pilbara: sail and teams to bulk carriers and bitumen. Hesperian Press: Carlisle, Australia.

Gregory, A.C. and Gregory, F.T. (1884). Journals of Australian explorations. Government Printer: Brisbane, Australia.

Grice, A.C. (2006). The impacts of invasive plant species on the biodiversity of Australian rangelands. The Rangeland Journal 28: 27-35.

Grice, A. and Martin, T.G. (2006). The Management of weeds and their impact on biodiversity in rangelands. CRC for Australian Weed Management: Townsville, Australia.

Groves, R.H. (Convenor), Hosking, J.R., Batianoff, G.N., Cooke, D.A., Cowie, I.D., Johnson, R.W., Keighery, G.J., Lepschi, B.J., Mitchell, A.A., Moerkerk, M., Randall, R.P., Rozefelds, A.C., Walsh N.G., and Waterhouse, B.M. (2003). Weed categories for natural and agricultural ecosystem management. Bureau of Rural
Sciences: Canberra, Australia.

Hennig, P. (2004). A brief history of land use (pp. 13-18). In: Van Vreeswyk, A.M.E., Payne, A.L., Leighton, K.A. and Hennig, P. (eds), An inventory and condition survey of the Pilbara Region, Western Australia. Technical Bulletin No. 92. Western Australian Department of Agriculture: Perth, Australia.

Hussey, B.M.J., Keighery, G.J., Dodd, J., Lloyd, S.G. and Cousens, R.D. (2007). Western weeds: a guide to the weeds of Western Australia. 2nd edn. The Weeds Society of WA: Victoria Park, Australia.

Keighery, G. and Keighery, B. (2008). Less weeds, more natives: the Oxalis corniculata complex in Western Australia. Wildflower Society of Western Australia Newsletter 46 (1): 31-32.

Keighery, G.J. and Longman, V. (2004). The naturalized vascular plants of Western Australia 1: Checklist, environmental weeds and distribution in IBRA regions. Plant Protection Quarterly 19: 12-32.

Kendrick, P.G. (2005). Feral palms. Landscope 20: 46-51.

Kodela, P.G. and Tindale, M.D. (2001). Acacia farnesiana. Flora of Australia 11A: 200, 205, 207. Australian Biological Resources Study: Canberra; CSIRO Publishing: Melbourne, Australia.

Land and Water Australia (2008a). Quantifying costs and benefits of Buffel Grass. Available at http:/ /lwa.gov.au/ node/2585 [accessed 13 April 2010].

Land and Water Australia (2008b). Optimising management of core mesquite infestations in Australia. Available at http://lwa.gov.au/node/2585 [accessed 13 April 2010].

Lazarides, M., Cowley, K. and Hohnen, P. (1997). CSIRO handbook of Australian weeds. CSIRO Publishing: Melbourne, Australia.

Longbottom, A. (2004). Declared plants and animals (pp. 53-57). In: Van Vreeswyk, A.M.E., Payne, A.L., Leighton, K.A. and Hennig, P. (eds), An inventory and condition survey of the Pilbara Region, Western Australia. Technical Bulletin No. 92. Western Australian Department of Agriculture: Perth, Australia.

McKenzie, N.L., van Leeuwen, S. and Pinder, A.M. (2009). Introduction to the Pilbara biodiversity survey. Records of the Western Australian Museum, Supplement 78: 3-89.

Martin, T.G., Campbell, S. and Grounds, S. (2006). Weeds of Australian rangelands. The Rangeland Journal 28: 3-26.

Mattiske, E. and Associates (1992). Flora and vegetation: proposed Marandoo Project area. Report prepared for Hamersley Iron, Australia.

Mattiske, E. and Associates (1995). Flora and vegetation: proposed Yandicoogina Junction Area. Report prepared for Hamersley Iron, Australia.

Morrison, S.M. and Scott, J.K. (1996a). Variation in populations of Tribulus terrestris (Zygophyllaceae), 2, chromosome numbers. Australian Journal of Botany 44: 191-199.

Morrison, S.M. and Scott, J.K. (1996b). Variation in populations of Tribulus terrestris (Zygophyllaceae), 3, izosyme analysis. Australian Journal of Botany 44: 201-212.

Mueller, F. (1886). Descriptions of new Australian plants. 
Victorian Naturalist 3: 108-110.

Petheram, R.J. and Kok, B. (1983). Plants of the Kimberley region of Western Australia. University of Western Australia Press: Nedlands, Australia.

Powell, A.M. (1978). Systematics of Flaveria (FlaveriinaeAsteraceae). Annals of the Missouri Botanical Garden 65: 590-636.

Randall, R.P. (2007). The introduced flora of Australia and its weed status. CRC for Australian Weed Management: Adelaide, Australia.

Scott, J.K. and Morrison, S.M. (1996). Variation in populations of Tribulus terrestris (Zygophyllaceae),1, burr morphology. Australian Journal of Botany 44: 175-190.

Storrs, M.J. and Lonsdale, M.W. (1995). Developing a weed management strategy for a conservation area (pp. 182-190). In: Proceedings of the Wet-Dry Tropics Wetland Management Workshop, 22-24 March, 1995, Jabiru, Northern Territory. Office of the Supervising Scientist: Canberra, Australia.

Strategen (2006). Yandicoogina junction SE Project: weed management plan. Strategen for Hamersley Iron, Pilbara Iron: Perth, Australia. van Klinken, R.D., Shepherd, D., Parr, R., Robinson, T.P. and Anderson, L. (2007). Mapping mesquite (Prosopis) distribution and density using aerial surveys. Rangeland Ecology and Management 60: 408-416.

Van Vreeswyk, A.M.E. (2004). Vegetation (pp. 109-124). In: Van Vreeswyk, A.M.E., Payne, A.L., Leighton, K.A. and Hennig, P. (eds), An inventory and condition survey of the Pilbara Region, Western Australia. Technical Bulletin No. 92. Western Australian Department of Agriculture: Perth, Australia.

Van Vreeswyk, A.M.E., Payne, A.L., Leighton, K.A. and Hennig, P. (eds) (2004). An inventory and condition survey of the Pilbara Region, Western Australia. Technical Bulletin No. 92. Western Australian Department of Agriculture: Perth, Australia.

Western Australian Herbarium (1998-). FloraBase-The Western Australian Flora. Department of Environment and Conservation. http://florabase.dec.wa.gov.au [accessed 20 April 2010].

Withnell, N. (1936). Early days in Roebourne. Self published: Perth, Australia.

Withnell Taylor, N.E. (2002). A saga of the North-West; Yerra-Muk-A Doo. Hesperian Press: Carlisle, Australia.

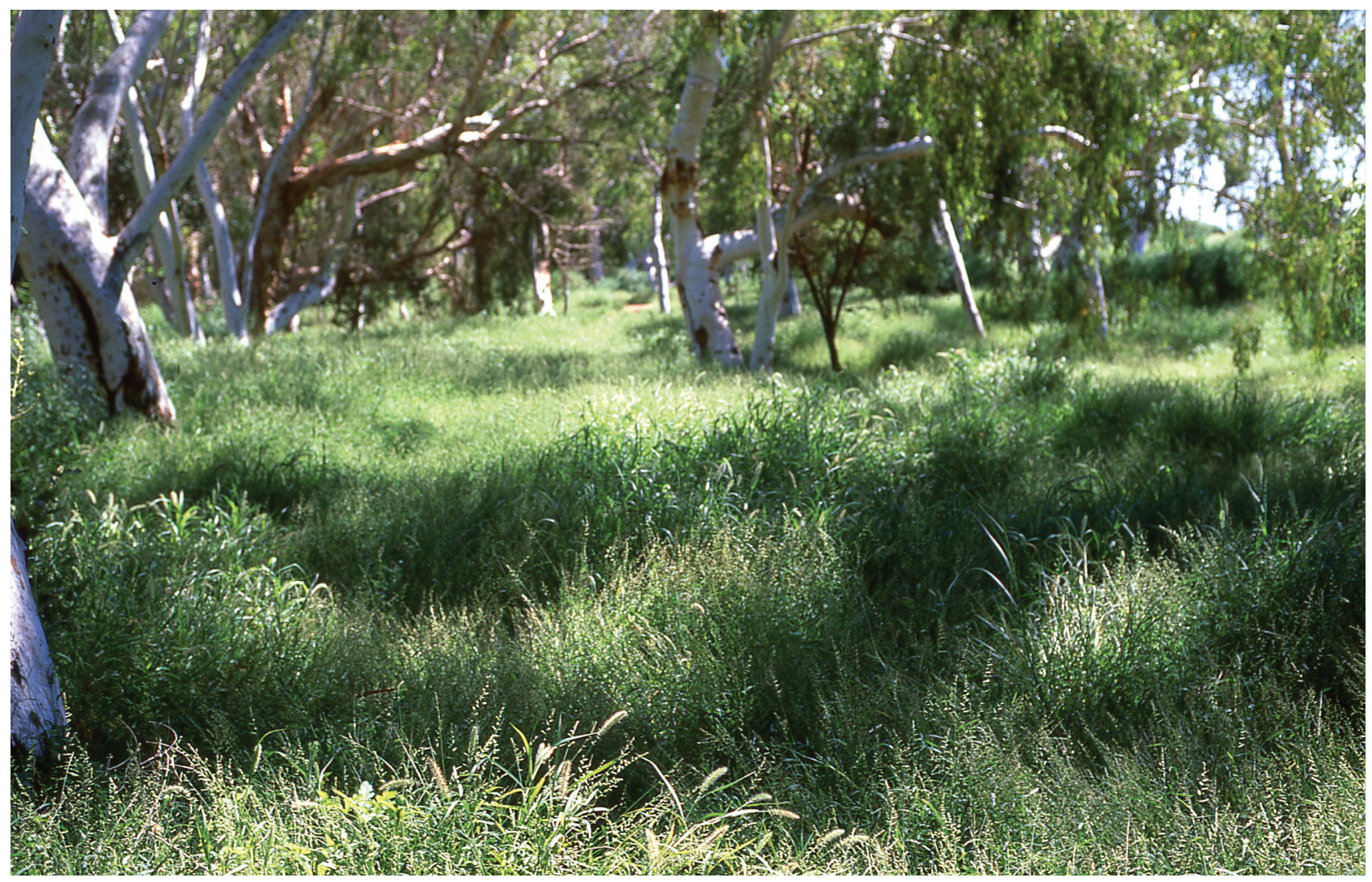

A understorey monoculture of Birdwood Grass (Cenchrus setiger), an environmental weed, on a bank of the De Grey River (G.J. Keighery). 